S. K. MOHANTA, R. KaR

\title{
SOME NEW COINCIDENCE POINT RESULTS FOR SINGLE-VALUED AND MULTI-VALUED MAPPINGS IN $b$-METRIC SPACES VIA DIGRAPHS
}

\begin{abstract}
S. K. Mohanta ${ }^{1}, \mathrm{R} . \mathrm{Kar}^{2}$. Some new coincidence point results for single-valued and multi-valued mappings in b-metric spaces via digraphs, Mat. Stud. 53 (2020), 69-84.

We introduce the concept of generalized $F-G$-contraction and prove some new coincidence point results for single-valued and multi-valued mappings in $b$-metric spaces endowed with a digraph $G$. Our results generalize and extend several well-known comparable results including Nadler's fixed point theorem for multi-valued mappings. Moreover, we give some examples to justify the validity of our main result.
\end{abstract}

1. Introduction. It is well-known that Banach contraction principle [6] is one of the most important theorems in classical functional analysis. Because of its simplicity and usefulness it has become a popular tool for solving existence and uniqueness problems in nonlinear analysis. Indeed, it is widely considered as the source of metric fixed point theory. Several authors successfully extended this interesting result in many directions. The study finds applications in different branches of mathematics and applied sciences such as variational and linear inequalities, optimal control problems, operation research, integral equations etc.. In 1969, Nadler [28] proved that every multi-valued contraction on a complete metric space has a fixed point. Since then, many authors including Gordji [21], Berinde [4], Pathak [29] and others studied lots of different types of fixed point theorems for multi-valued contractions. In 2012, Wardowski [31] introduced the concept of $F$-contraction for single-valued mappings and studied fixed points for such class of mappings in metric spaces. By using Wardowski's [31] and Nadler's [28] ideas, many authors (see [1, 2, 15, 24] and references therein) studied fixed points for multi-valued mappings.

In 1989, Bakhtin [5] introduced the concept of $b$-metric spaces as a generalization of metric spaces and generalized the famous Banach contraction principle in metric spaces to $b$ metric spaces. In recent investigations, the study of fixed point theory combining a graph is a new development in the domain of contractive type multi-valued theory. Starting from these considerations, the study of fixed points and common fixed points of mappings satisfying a certain contractive type condition endowed with a graph attracted many researchers, see for examples $[8,9,10,17,18,23,26,30]$. Inspired and motivated by the results in $[15,31]$, we introduce the concept of generalized $F$ - $G$-contraction in $b$-metric spaces and obtain some coincidence point results for hybrid pair of single-valued and multi-valued mappings in $b$ metric spaces with a digraph. Our main result extends Nadler's fixed point theorem in the

2020 Mathematics Subject Classification: 47H10, 54H25.

Keywords: b-metric; digraph; generalized F-G-contractions; coincidence point. doi:10.30970/ms.53.1.69-84

(C) S. K. Mohanta, R. Kar, 2020 
setting of $b$-metric spaces. Finally, we give some examples to justify the validity of our main result.

2. Some basic concepts. In this section, we recall some basic known definitions, notations and results in $b$-metric spaces which will be used in the sequel. Throughout this article, $\mathbb{N}, \mathbb{R}, \mathbb{R}^{+}$denote the set of natural numbers, the set of real numbers and the set of positive real numbers, respectively.

Definition 1 ([12]). Let $X$ be a nonempty set and $s \geq 1$ be a given real number. A function $d: X \times X \rightarrow[0, \infty)$ is said to be a $b$-metric on $X$ if the following conditions hold:

(i) $d(x, y)=0$ if and only if $x=y$;

(ii) $d(x, y)=d(y, x)$ for all $x, y \in X$;

(iii) $d(x, y) \leq s(d(x, z)+d(z, y))$ for all $x, y, z \in X$.

The pair $(X, d)$ is called a $b$-metric space.

It is worth mentioning that the class of $b$-metric spaces is effectively larger than that of the ordinary metric spaces. The following example illustrates the above fact.

Example 1. Let $X=\mathbb{R}$. Define $d: X \times X \rightarrow[0, \infty)$ by $d(x, y)=|x-y|^{2}$ for all $x, y \in X$. Then $(X, d)$ is a $b$-metric space with the coefficient $s=2$, but it is not a metric space since the triangle inequality does not hold. Indeed, we have

$$
d(-1,0)+d(0,1)=1+1=2<4=d(-1,1) .
$$

Example $2([3])$. Let $p \in(0,1)$. Then the set

$$
l^{p}(\mathbb{R}):=\left\{\left(x_{n}\right) \subseteq \mathbb{R}: \sum_{n=1}^{\infty}\left|x_{n}\right|^{p}<\infty\right\}
$$

endowed with the functional $d: l^{p}(\mathbb{R}) \times l^{p}(\mathbb{R}) \rightarrow \mathbb{R}$ given by

$$
d\left(\left(x_{n}\right),\left(y_{n}\right)\right)=\left(\sum_{n=1}^{\infty}\left|x_{n}-y_{n}\right|^{p}\right)^{\frac{1}{p}}
$$

for all $\left(x_{n}\right),\left(y_{n}\right) \in l^{p}(\mathbb{R})$ is a $b$-metric space with $s=2^{\frac{1}{p}}$.

Definition 2 ([11]). Let $(X, d)$ be a $b$-metric space, $x \in X$ and $\left(x_{n}\right)$ be a sequence in $X$. Then

(i) $\left(x_{n}\right)$ converges to $x$ if and only if $\lim _{n \rightarrow \infty} d\left(x_{n}, x\right)=0$. We denote this by $\lim _{n \rightarrow \infty} x_{n}=x$ or $x_{n} \rightarrow x(n \rightarrow \infty)$.

(ii) $\left(x_{n}\right)$ is Cauchy if and only if $\lim _{n, m \rightarrow \infty} d\left(x_{n}, x_{m}\right)=0$.

(iii) $(X, d)$ is complete if and only if every Cauchy sequence in $X$ is convergent.

Remark 1 ([11]). In a $b$-metric space $(X, d)$, the following assertions hold:

(i) A convergent sequence has a unique limit.

(ii) Each convergent sequence is Cauchy. 
(iii) In general, a $b$-metric is not continuous.

Definition 3 ([22]). Let $(X, d)$ be a $b$-metric space. A subset $A \subseteq X$ is said to be open if and only if for any $a \in A$, there exists $\epsilon>0$ such that the open ball $B(a, \epsilon) \subseteq A$. The family of all open subsets of $X$ will be denoted by $\tau$.

Theorem 1 ([22]). $\tau$ defines a topology on $(X, d)$.

Theorem 2 ([22]). Let $(X, d)$ be a $b$-metric space and $\tau$ be the topology defined above. Then for any nonempty subset $A \subseteq X$ we have

(i) $A$ is closed if and only if for any sequence $\left(x_{n}\right)$ in $A$ which converges to $x$, we have $x \in A$;

(ii) if we define $\bar{A}$ to be the intersection of all closed subsets of $X$ which contains $A$, then for any $x \in \bar{A}$ and for any $\epsilon>0$, we have $B(x, \epsilon) \cap A \neq \varnothing$.

Definition $4([27])$. Let $(X, d)$ be a $b$-metric space and $A$ be a nonempty subset of $X$. The diameter of $A$, denoted by $\delta(A)$, is defined by $\delta(A)=\sup \{d(x, y): x, y \in A\}$. The subset $A$ is said to be bounded if $\delta(A)$ is finite.

Let $(X, d)$ be a $b$-metric space and $C B(X)$ be the set of all nonempty closed bounded subsets of $X$. An element $x \in X$ is said to be a fixed point of a multi-valued mapping $T: X \rightarrow 2^{X}$ if $x \in T x$, where $2^{X}$ denotes the collection of all nonempty subsets of $X$. For $A, B \in C B(X)$, define

$$
H(A, B)=\max \left\{\sup _{x \in A} d(x, B), \sup _{y \in B} d(y, A)\right\}
$$

where $d(x, B)=\inf \{d(x, y): y \in B\}$. Such a map $H$ is called the Hausdorff $b$-metric induced by the $b$-metric $d$.

We now present some lemmas which can be found in $[12,13,14]$.

Lemma 1. Let $(X, d)$ be a $b$-metric space with the coefficient $s \geq 1$. For any $A, B, C \in$ $C B(X)$ and any $x, y \in X$, we have the following:

(i) $d(x, B) \leq d(x, b)$ for any $b \in B$;

(ii) $d(x, B) \leq H(A, B)$ for any $x \in A$;

(iii) $d(x, A) \leq s[d(x, y)+d(y, A)]$.

Lemma 2. Let $(X, d)$ be a $b$-metric space with the coefficient $s \geq 1$ and $A, B \in C B(X)$. Then, for each $h>1$ and for each $a \in A$, there exists $b(a) \in B$ such that $d(a, b(a)) \leq$ $h H(A, B)$.

Lemma 3. Let $(X, d)$ be a $b$-metric space with the coefficient $s \geq 1$. For $A \in C B(X)$ and $x \in X$, we have

$$
d(x, A)=0 \Longleftrightarrow x \in \bar{A}=A,
$$

where $\bar{A}$ denotes the closure of the set $A$. 
Let $(X, d)$ be a $b$-metric space with the coefficient $s \geq 1$ and $\rho$ be a binary relation over $X$. Denote $S=\rho \cup \rho^{-1}$. Then

$$
x S y \Leftrightarrow x \rho y \text { and } y \rho x \text { for any } x, y \in X .
$$

In fact, $x S y \Rightarrow y S x$ for all $x, y \in X$.

Definition 5. A symmetrical relation $S$ is regular in $(X, d)$ if the following condition holds:

If the sequence $\left(x_{n}\right)$ in $X$ and the point $x \in X$ are such that $x_{n} S x_{n+1}$ for all $n \geq 1$ and $\lim _{n \rightarrow \infty} d\left(x_{n}, x\right)=0$, then there exists a subsequence $\left(x_{n_{i}}\right)$ of $\left(x_{n}\right)$ such that $x_{n_{i}} S x$ for all $i \geq 1$.

Definition 6. Let $(X, d)$ be a $b$-metric space and $\rho$ be a binary relation over $X$. Then the mapping $f: X \rightarrow X$ is called $S$-preserving if $f$ maps comparable elements into comparable elements, that is,

$$
x, y \in X, x S y \Rightarrow(f x) S(f y) .
$$

For subsets $A, B$ of $X$, we use the following notation:

$$
A S B \Leftrightarrow a S b \text { for all } a \in A, b \in B .
$$

Definition 7. Let $(X, d)$ be a $b$-metric space and $\rho$ be a binary relation over $X$. Then the mapping $T: X \rightarrow C B(X)$ is called $S$-preserving if

$$
\forall x, y \in X, x S y \Rightarrow(T x) S(T y) .
$$

Definition 8 ([27]). Let $(X, d)$ be a $b$-metric space and $T: X \rightarrow C B(X)$ and $g: X \rightarrow X$ be two mappings. If $y=g x \in T x$ for some $x$ in $X$, then $x$ is called a coincidence point of $T$ and $g$ and $y$ is called a point of coincidence of $T$ and $g$.

We next review some basic notions in graph theory.

Let $(X, d)$ be a $b$-metric space. We assume that $G$ is a digraph with the set of vertices $V(G)=X$ and the set $E(G)$ of its edges contains all the loops, i.e., $\Delta \subseteq E(G)$ where $\Delta=\{(x, x): x \in X\}$. We also assume that $G$ has no parallel edges and obtain a weighted graph by assigning to each edge the distance between its vertices. We can identify $G$ with the pair $(V(G), E(G))$. We denote the conversion of a graph $G$ by $G^{-1}$, that is, the graph obtained from $G$ by reversing the direction of the edges i.e., $E\left(G^{-1}\right)=\{(x, y) \in X \times X:(y, x) \in$ $E(G)\}$. Let $\tilde{G}$ denote the undirected graph obtained from $G$ by ignoring the direction of edges. Actually, it will be more convenient for us to treat $\tilde{G}$ as a digraph for which the set of its edges is symmetric. Under this convention,

$$
E(\tilde{G})=E(G) \cup E\left(G^{-1}\right) .
$$

Our graph theory notations and terminology are standard and can be found in all graph theory books, like $[7,16,20]$. If $x, y$ are vertices of the digraph $G$, then a path in $G$ from $x$ to $y$ of length $n(n \in \mathbb{N})$ is a sequence $\left(x_{i}\right)_{i=0}^{n}$ of $n+1$ vertices such that $x_{0}=x, x_{n}=y$ and $\left(x_{i-1}, x_{i}\right) \in E(G)$ for $i=1,2, \cdots, n$. A graph $G$ is connected if there is a path between any two vertices of $G$. $G$ is weakly connected if $\tilde{G}$ is connected.

Definition 9. Let $(X, d)$ be a $b$-metric space with the coefficient $s \geq 1$ and let $G=$ $(V(G), E(G))$ be a graph. Then the mapping $f: X \rightarrow X$ is called edge preserving if

$$
x, y \in X,(x, y) \in E(\tilde{G}) \Rightarrow(f x, f y) \in E(\tilde{G}) .
$$


Definition 10. Let $(X, d)$ be a $b$-metric space with a graph $G=(V(G), E(G))$ and let $f, g: X \rightarrow X$ be two mappings. Then $f$ is called edge preserving w.r.t. $g$ if

$$
x, y \in X,(g x, g y) \in E(\tilde{G}) \Rightarrow(f x, f y) \in E(\tilde{G}) .
$$

Definition 11. Let $(X, d)$ be a $b$-metric space with a graph $G=(V(G), E(G))$. Then the mapping $T: X \rightarrow C B(X)$ is called edge preserving if

$$
x, y \in X, x \neq y,(x, y) \in E(\tilde{G}) \Rightarrow\left(z_{1}, z_{2}\right) \in E(\tilde{G}) \text {, for all } z_{1} \in T x, z_{2} \in T y .
$$

Definition 12. Let $(X, d)$ be a $b$-metric space with a graph $G=(V(G), E(G))$. Let $T: X \rightarrow$ $C B(X)$ be a multi-valued mapping and $g: X \rightarrow X$ be a single-valued mapping. Then $T$ is called edge preserving w.r.t. $g$ if

$$
x, y \in X, x \neq y,(g x, g y) \in E(\tilde{G}) \Rightarrow\left(z_{1}, z_{2}\right) \in E(\tilde{G}) \text {, for all } z_{1} \in T x, z_{2} \in T y .
$$

Definition 13 ([15]). Let $s \geq 1$ be a real number. We denote by $\mathbb{F}_{s}$ the family of all functions $F: \mathbb{R}^{+} \rightarrow \mathbb{R}$ with the following properties:

(F1) $F$ is strictly increasing;

(F2) for each sequence $\left(\alpha_{n}\right)$ of positive numbers, $\lim _{n \rightarrow \infty} \alpha_{n}=0$ if and only if $\lim _{n \rightarrow \infty} F\left(\alpha_{n}\right)=-\infty$;

(F3) for each sequence $\left(\alpha_{n}\right)$ of positive numbers with $\lim _{n \rightarrow \infty} \alpha_{n}=0$, there exists $k \in(0,1)$ such that $\lim _{n \rightarrow \infty}\left(\alpha_{n}\right)^{k} F\left(\alpha_{n}\right)=0$;

(F4) for each sequence $\left(\alpha_{n}\right)$ of positive numbers such that $\tau+F\left(s \alpha_{n}\right) \leq F\left(\alpha_{n-1}\right)$ for all $n \in \mathbb{N}$ and some $\tau>0$, then $\tau+F\left(s^{n} \alpha_{n}\right) \leq F\left(s^{n-1} \alpha_{n-1}\right)$ for all $n \in \mathbb{N}$.

Example 3 ([15]). If $F(x)=x+\ln x, x>0$, then $F \in \mathbb{F}_{s}$.

Example 4 ([15]). If $F(x)=\ln x, x>0$, then $F \in \mathbb{F}_{s}$.

Definition $14([15])$. Let $(X, d)$ be a $b$-metric space with the coefficient $s \geq 1$. A multivalued mapping $T: X \rightarrow C B(X)$ is called an $F$-contraction of Nadler type if there exist $F \in \mathbb{F}_{s}, \tau>0$ such that

$$
2 \tau+F(s H(T x, T y)) \leq F(d(x, y)),
$$

for all $x, y \in X$ with $T x \neq T y$.

3. Main Results. We begin this section by introducing the following definition.

Definition 15. Let $(X, d)$ be a $b$-metric space with the coefficient $s \geq 1$ and let $G=$ $(V(G), E(G))$ be a digraph. Then the pair $(T, f)$ of mappings $T: X \rightarrow C B(X)$ and $f: X \rightarrow$ $X$ is called a generalized $F$-G-contraction of Nadler type if there exist $F \in \mathbb{F}_{s}$ and $\tau>0$ such that

$$
2 \tau+F(s H(T x, T y)) \leq F\left(M_{s}(f x, f y)\right),
$$

for all $x, y \in X$ with $T x \neq T y$ and $(f x, f y) \in E(\tilde{G})$ where

$$
M_{s}(f x, f y)=\max \left\{d(f x, f y), d(f x, T x), \frac{d(f y, T y)}{2 s}, \frac{d(f x, T y)+d(f y, T x)}{2 s}\right\} .
$$


Actually, $M_{s}(f x, f y)$ does not depend only from $s, f x$ and $f y$. It depends from $x, y$ and $T$ too. But we use this notation for the simplicity.

We now assume that $(X, d)$ is a $b$-metric space endowed with a reflexive digraph $G$ such that $V(G)=X$ and $G$ has no parallel edges. Let $f: X \rightarrow X$ and $T: X \rightarrow C B(X)$ be such that $T(X) \subseteq f(X)$. Let $x_{0} \in X$ be arbitrary. Since $T(X) \subseteq f(X)$, there exists an element $x_{1} \in X$ such that $f x_{1} \in T x_{0}$. Continuing in this way, we can construct a sequence $\left(f x_{n}\right)$ such that $f x_{n} \in T x_{n-1}, n=1,2,3, \cdots$.

Our main result is as follows:

Theorem 3. Let $(X, d)$ be a $b$-metric space with the coefficient $s \geq 1$ and let $G=$ $(V(G), E(G))$ be a graph. Let $T: X \rightarrow C B(X)$ and $f: X \rightarrow X$ be such that $T(X) \subseteq f(X)$ and $f(X)$ a complete subspace of $X$. Assume that $T$ is edge preserving w.r.t. $f$ and there exist a function $F \in \mathbb{F}_{s}$ which is continuous from right and $\tau>0$ such that $(T, f)$ is generalized F-G-contraction of Nadler type. Suppose also that the triple $(X, d, G)$ has the following property:

$(*)$ If $\left(f x_{n}\right)$ is a sequence in $X$ such that $f x_{n} \rightarrow x$ and $\left(f x_{n}, f x_{n+1}\right) \in E(\tilde{G})$ for all $n \geq 1$, then there exists a subsequence $\left(f x_{n_{i}}\right)$ of $\left(f x_{n}\right)$ such that $\left(f x_{n_{i}}, x\right) \in E(\tilde{G})$ for all $i \geq 1$.

If there exists $x_{0} \in X$ such that $\left(f x_{0}, z\right) \in E(\tilde{G})$ for some $z \in T x_{0}$, then $f$ and $T$ have a point of coincidence in $f(X)$.

Proof. Suppose there exists $x_{0} \in X$ such that $\left(f x_{0}, z\right) \in E(\tilde{G})$ for some $z \in T x_{0}$. If $f x_{0} \in$ $T x_{0}$, then there is nothing to prove. So, we assume that $f x_{0} \notin T x_{0}$. This ensures that $d\left(f x_{0}, T x_{0}\right)>0$, since $T x_{0}$ is closed. Therefore, $d\left(f x_{0}, y\right)>0$ for all $y \in T x_{0}$. As $T x_{0} \subseteq$ $f(X)$ is nonempty, there exists $x_{1} \in X$ such that $z=f x_{1} \in T x_{0}, d\left(f x_{0}, f x_{1}\right)>0$ and $\left(f x_{0}, f x_{1}\right) \in E(\tilde{G})$. If $f x_{1} \in T x_{1}$, then $f$ and $T$ have a point of coincidence in $f(X)$. So, we assume that $f x_{1} \notin T x_{1}$ and hence $T x_{0} \neq T x_{1}$ which gives that $x_{0} \neq x_{1}$. Since $F \in \mathbb{F}_{s}$ is continuous from the right, there exists $h>1$ such that

$$
F\left(h s H\left(T x_{0}, T x_{1}\right)\right)<F\left(s H\left(T x_{0}, T x_{1}\right)\right)+\tau .
$$

As $f x_{1} \in T x_{0}$ and $h>1$, by applying Lemma 2, there exists $f x_{2} \in T x_{1}$ for some $x_{2} \in X$ such that

$$
d\left(f x_{1}, f x_{2}\right) \leq h H\left(T x_{0}, T x_{1}\right) .
$$

Since $f x_{1} \notin T x_{1}$, we have $d\left(f x_{1}, T x_{1}\right)>0$ and consequently, $d\left(f x_{1}, f x_{2}\right)>0$.

By using monotonicity property of $F$, we obtain from conditions (2) and (3) that

$$
F\left(s d\left(f x_{1}, f x_{2}\right)\right) \leq F\left(h s H\left(T x_{0}, T x_{1}\right)\right)<F\left(s H\left(T x_{0}, T x_{1}\right)\right)+\tau .
$$

By using conditions (1) and (4), we get

$$
2 \tau+F\left(s d\left(f x_{1}, f x_{2}\right)\right)<2 \tau+F\left(s H\left(T x_{0}, T x_{1}\right)\right)+\tau \leq F\left(M_{s}\left(f x_{0}, f x_{1}\right)\right)+\tau .
$$

Therefore,

$$
\tau+F\left(s d\left(f x_{1}, f x_{2}\right)\right)<F\left(M_{s}\left(f x_{0}, f x_{1}\right)\right) .
$$

As $T$ is edge preserving w.r.t. $f$ and $x_{0} \neq x_{1},\left(f x_{0}, f x_{1}\right) \in E(\tilde{G}), f x_{1} \in T x_{0}, f x_{2} \in T x_{1}$, it follows that $\left(f x_{1}, f x_{2}\right) \in E(\tilde{G})$. If $f x_{2} \in T x_{2}$, then the theorem is proved. So, we assume that $f x_{2} \notin T x_{2}$. As a consequence, it follows that $T x_{1} \neq T x_{2}$ and this implies that $x_{1} \neq x_{2}$. 
By an argument similar to that used above, there exists $f x_{3} \in T x_{2}$ for some $x_{3} \in X$ and $d\left(f x_{2}, f x_{3}\right)>0$ such that

$$
\tau+F\left(s d\left(f x_{2}, f x_{3}\right)\right)<F\left(M_{s}\left(f x_{1}, f x_{2}\right)\right) .
$$

As $T$ is edge preserving w.r.t. $f$ and $x_{1} \neq x_{2},\left(f x_{1}, f x_{2}\right) \in E(\tilde{G}), f x_{2} \in T x_{1}, f x_{3} \in T x_{2}$, it follows that $\left(f x_{2}, f x_{3}\right) \in E(\tilde{G})$. Continuing this process, we can construct a sequence $\left(f x_{n}\right)$ in $f(X)$ such that $f x_{n} \in T x_{n-1}, f x_{n} \notin T x_{n}, d\left(f x_{n}, f x_{n+1}\right)>0,\left(f x_{n}, f x_{n+1}\right) \in E(\tilde{G})$ for $n=0,1,2, \cdots$ and

$$
\tau+F\left(s d\left(f x_{n}, f x_{n+1}\right)\right)<F\left(M_{s}\left(f x_{n-1}, f x_{n}\right)\right), \text { for all } n \in \mathbb{N} .
$$

This gives that

$$
F\left(s d\left(f x_{n}, f x_{n+1}\right)\right)<F\left(M_{s}\left(f x_{n-1}, f x_{n}\right)\right) \text {, for all } n \in \mathbb{N} .
$$

$F$ being strictly increasing, it follows that

$$
0<s d\left(f x_{n}, f x_{n+1}\right)<M_{s}\left(f x_{n-1}, f x_{n}\right) \text {, for all } n \in \mathbb{N} .
$$

This implies that

$$
d\left(f x_{n}, f x_{n+1}\right)<M_{s}\left(f x_{n-1}, f x_{n}\right) \text {, for all } n \in \mathbb{N} \text {. }
$$

Here,

$$
M_{s}\left(f x_{n-1}, f x_{n}\right)=\max \left\{\begin{array}{l}
d\left(f x_{n-1}, f x_{n}\right), d\left(f x_{n-1}, T x_{n-1}\right), \frac{d\left(f x_{n}, T x_{n}\right)}{2 s}, \\
\frac{d\left(f x_{n-1}, T x_{n}\right)+d\left(f x_{n}, T x_{n-1}\right)}{2 s}
\end{array}\right\} .
$$

We now estimate each of the expressions on the right hand side of condition (7) separately.

$$
\begin{gathered}
d\left(f x_{n-1}, T x_{n-1}\right) \leq d\left(f x_{n-1}, f x_{n}\right), \text { as } f x_{n} \in T x_{n-1}, \\
\frac{d\left(f x_{n}, T x_{n}\right)}{2 s} \leq \frac{d\left(f x_{n}, f x_{n+1}\right)}{2 s}<d\left(f x_{n}, f x_{n+1}\right) \text {, as } f x_{n+1} \in T x_{n}, \\
\frac{d\left(f x_{n-1}, T x_{n}\right)+d\left(f x_{n}, T x_{n-1}\right)}{2 s} \leq \frac{d\left(f x_{n-1}, f x_{n+1}\right)}{2 s} \leq \\
\leq \frac{d\left(f x_{n-1}, f x_{n}\right)+d\left(f x_{n}, f x_{n+1}\right)}{2} \text {, as } f x_{n} \in T x_{n-1} .
\end{gathered}
$$

Therefore,

$$
\begin{gathered}
M_{s}\left(f x_{n-1}, f x_{n}\right)=\max \left\{\begin{array}{l}
d\left(f x_{n-1}, f x_{n}\right), d\left(f x_{n-1}, T x_{n-1}\right), \frac{d\left(f x_{n}, T x_{n}\right)}{2 s}, \\
\frac{d\left(f x_{n-1}, T x_{n}\right)+d\left(f x_{n}, T x_{n-1}\right)}{2 s}
\end{array}\right\} \leq \\
\leq \max \left\{\begin{array}{l}
d\left(f x_{n-1}, f x_{n}\right), d\left(f x_{n}, f x_{n+1}\right), \\
\frac{d\left(f x_{n-1}, f x_{n}\right)+d\left(f x_{n}, f x_{n+1}\right)}{2}
\end{array}\right\}=\max \left\{d\left(f x_{n-1}, f x_{n}\right), d\left(f x_{n}, f x_{n+1}\right)\right\} .
\end{gathered}
$$

If $\max \left\{d\left(f x_{n-1}, f x_{n}\right), d\left(f x_{n}, f x_{n+1}\right)\right\}=d\left(f x_{n}, f x_{n+1}\right)$, then $M_{s}\left(f x_{n-1}, f x_{n}\right) \leq d\left(f x_{n}, f x_{n+1}\right)$, which contradicts condition (6).

Therefore, $\max \left\{d\left(f x_{n-1}, f x_{n}\right), d\left(f x_{n}, f x_{n+1}\right)\right\}=d\left(f x_{n-1}, f x_{n}\right)$ and hence

$$
M_{s}\left(f x_{n-1}, f x_{n}\right) \leq d\left(f x_{n-1}, f x_{n}\right) \text {, for all } n \in \mathbb{N} .
$$


So, condition (5) implies that

$$
\tau+F\left(s d\left(f x_{n}, f x_{n+1}\right)\right)<F\left(d\left(f x_{n-1}, f x_{n}\right)\right) \text {, for all } n \in \mathbb{N} .
$$

Let us put $\alpha_{n}=d\left(f x_{n}, f x_{n+1}\right)>0$ for all $n \in \mathbb{N} \cup\{0\}$. By property (F4), we obtain from condition (8) that $\tau+F\left(s^{n} \alpha_{n}\right) \leq F\left(s^{n-1} \alpha_{n-1}\right)$, for all $n \in \mathbb{N}$. This gives that

$$
F\left(s^{n} \alpha_{n}\right) \leq F\left(s^{n-1} \alpha_{n-1}\right)-\tau, \text { for all } n \in \mathbb{N} .
$$

By repeated use of condition (9), we get

$$
F\left(s^{n} \alpha_{n}\right) \leq F\left(s^{n-1} \alpha_{n-1}\right)-\tau \leq \cdots \leq F\left(\alpha_{0}\right)-n \tau, \text { for all } n \in \mathbb{N} .
$$

Since $\tau>0$, we have $\lim _{n \rightarrow \infty} F\left(s^{n} \alpha_{n}\right)=-\infty$. By applying property $(F 2)$, we get $\lim _{n \rightarrow \infty} s^{n} \alpha_{n}=0$. By property $(F 3)$, there exists $k \in(0,1)$ such that

$$
\lim _{n \rightarrow \infty}\left(s^{n} \alpha_{n}\right)^{k} F\left(s^{n} \alpha_{n}\right)=0 .
$$

From condition (10), we get

$$
\left(s^{n} \alpha_{n}\right)^{k} F\left(s^{n} \alpha_{n}\right)-\left(s^{n} \alpha_{n}\right)^{k} F\left(\alpha_{0}\right) \leq-n \tau\left(s^{n} \alpha_{n}\right)^{k}<0, \text { for all } n \in \mathbb{N} .
$$

Taking limit as $n \rightarrow \infty$, we obtain

$$
\lim _{n \rightarrow \infty} n\left(s^{n} \alpha_{n}\right)^{k}=0
$$

It follows from condition (11) that there exists $n_{1} \in \mathbb{N}$ such that $n\left(s^{n} \alpha_{n}\right)^{k} \leq 1$ for all $n \geq n_{1}$. As a consequence, we have

$$
s^{n} \alpha_{n} \leq \frac{1}{n^{\frac{1}{k}}}, \text { for all } n \geq n_{1} .
$$

We now show that $\left(f x_{n}\right)$ is a Cauchy sequence in $f(X)$.

For $m, n \in \mathbb{N}$ with $m>n \geq n_{1}$, we obtain by using condition (12) that

$$
\begin{gathered}
d\left(f x_{n}, f x_{m}\right) \leq s d\left(f x_{n}, f x_{n+1}\right)+s^{2} d\left(f x_{n+1}, f x_{n+2}\right)+\cdots+ \\
+s^{m-n-1} d\left(f x_{m-2}, f x_{m-1}\right)+s^{m-n-1} d\left(f x_{m-1}, f x_{m}\right) \leq \\
\leq\left[s \alpha_{n}+s^{2} \alpha_{n+1}+\cdots+s^{m-n-1} \alpha_{m-2}+s^{m-n} \alpha_{m-1}\right]= \\
=\frac{1}{s^{n-1}}\left[s^{n} \alpha_{n}+s^{n+1} \alpha_{n+1}+\cdots+s^{m-2} \alpha_{m-2}+s^{m-1} \alpha_{m-1}\right]= \\
\quad=\frac{1}{s^{n-1}} \sum_{i=n}^{m-1} s^{i} \alpha_{i}<\frac{1}{s^{n-1}} \sum_{i=n}^{\infty} s^{i} \alpha_{i} \leq \frac{1}{s^{n-1}} \sum_{i=n}^{\infty} \frac{1}{i^{\frac{1}{k}}} .
\end{gathered}
$$

Since $\sum_{i=1}^{\infty} \frac{1}{i^{\frac{1}{k}}}<\infty$, it follows that

$$
\lim _{m, n \rightarrow \infty} d\left(f x_{n}, f x_{m}\right)=0 .
$$

This gives that $\left(f x_{n}\right)$ is a Cauchy sequence in $f(X)$. As $f(X)$ is complete, there exists $u \in f(X)$ such that $\lim _{n \rightarrow \infty} f x_{n}=u=f t$ for some $t \in X$. 
We now observe that if there exists a subsequence $\left(f x_{n_{k}}\right)$ of $\left(f x_{n}\right)$ such that $f x_{n_{k}} \in T t$ for all $k \in \mathbb{N}$, then $\lim _{k \rightarrow \infty} f x_{n_{k}}=f t \in T t, T t$ being closed. This shows that $f$ and $T$ have a point of coincidence in $f(X)$. Now we assume that there exists $n_{0} \in \mathbb{N}$ such that $f x_{n} \notin T t$ for all $n \in \mathbb{N}$ with $n \geq n_{0}$. This ensures that $f x_{n+1} \notin T t$ for all $n \geq n_{0}$ and hence $T x_{n} \neq T t$ for all $n \geq n_{0}$. Moreover, by property $(*)$, there exists a subsequence $\left(f x_{n_{i}}\right)$ of $\left(f x_{n}\right)$ such that $\left(f x_{n_{i}}, f t\right) \in E(\tilde{G})$ for all $i \in \mathbb{N}$. Consequently, it follows that $T x_{n_{i}} \neq T t$ for all $i \geq n_{0}$.

Using condition (1), we obtain

$$
2 \tau+F\left(s H\left(T x_{n_{i}}, T t\right)\right) \leq F\left(M_{s}\left(f x_{n_{i}}, f t\right)\right) \text {, for all } i \geq n_{0} .
$$

This gives that

$$
2 \tau+F\left(s d\left(f x_{n_{i}+1}, T t\right)\right) \leq 2 \tau+F\left(s H\left(T x_{n_{i}}, T t\right)\right) \leq F\left(M_{s}\left(f x_{n_{i}}, f t\right)\right),
$$

for all $i \geq n_{0}$. Since $\tau>0$, we get from condition (13) that

$$
F\left(s d\left(f x_{n_{i}+1}, T t\right)\right)<F\left(M_{s}\left(f x_{n_{i}}, f t\right)\right) \text {, for all } i \geq n_{0} .
$$

Since $F$ is strictly increasing, we have

$$
s d\left(f x_{n_{i}+1}, T t\right)<M_{s}\left(f x_{n_{i}}, f t\right) \text {, for all } i \geq n_{0} .
$$

Now, we shall show that

$$
\begin{gathered}
M_{s}\left(f x_{n_{i}}, f t\right)<\frac{3}{4} d(f t, T t), \text { where } \\
M_{s}\left(f x_{n_{i}}, f t\right)=\max \left\{\begin{array}{l}
d\left(f x_{n_{i}}, f t\right), d\left(f x_{n_{i}}, T x_{n_{i}}\right), \frac{d(f t, T t)}{2 s}, \\
\frac{d\left(f x_{n_{i}}, T t\right)+d\left(f t, T x_{n_{i}}\right)}{2 s}
\end{array}\right\} .
\end{gathered}
$$

Suppose that $d(f t, T t) \neq 0$. Let $\epsilon=\frac{d(f t, T t)}{4 s^{2}}>0$. Since $f x_{n_{i}} \rightarrow f t$, there exists $k_{1} \in \mathbb{N}$ such that

$$
d\left(f x_{n_{i}}, f t\right)<\frac{d(f t, T t)}{4 s^{2}}, \text { for each } i \geq k_{1} .
$$

As $f x_{n} \rightarrow f t$, there exists $k_{2} \in \mathbb{N}$ such that

$$
d\left(f x_{n_{i}+1}, f t\right)<\frac{d(f t, T t)}{4 s^{2}}, \text { for each } i \geq k_{2} .
$$

So, it must be the case that

$$
d\left(f t, T x_{n_{i}}\right) \leq d\left(f t, f x_{n_{i}+1}\right)<\frac{d(f t, T t)}{4 s^{2}}, \text { for each } i \geq k_{2}
$$

As $d\left(f x_{n_{i}}, T t\right) \leq s\left[d\left(f x_{n_{i}}, f t\right)+d(f t, T t)\right]$, it follows that

$$
d\left(f x_{n_{i}}, T t\right)<\frac{d(f t, T t)}{4 s}+s d(f t, T t) \leq \frac{5 s}{4} d(f t, T t), \text { for each } i \geq k_{1} .
$$

Put $k_{3}=\max \left\{k_{1}, k_{2}\right\}$. Then, for $i \geq k_{3}$, we have

$$
d\left(f x_{n_{i}}, T x_{n_{i}}\right) \leq d\left(f x_{n_{i}}, f x_{n_{i}+1}\right) \leq s\left[d\left(f x_{n_{i}}, f t\right)+d\left(f t, f x_{n_{i}+1}\right)\right]<\frac{d(f t, T t)}{2 s}
$$


and

$$
\frac{d\left(f x_{n_{i}}, T t\right)+d\left(f t, T x_{n_{i}}\right)}{2 s}<\frac{1}{2 s}\left(\frac{5 s}{4}+\frac{1}{4 s^{2}}\right) d(f t, T t) \leq \frac{1}{2 s}\left(\frac{5 s}{4}+\frac{s}{4}\right) d(f t, T t)=\frac{3}{4} d(f t, T t) .
$$

Now put $k=\max \left\{n_{0}, k_{3}\right\}$. Then, for $i \geq k$, it follows from conditions (15), (16), (17), (18) and (19) that

$$
M_{s}\left(f x_{n_{i}}, f t\right)<\frac{3}{4} d(f t, T t) .
$$

Therefore, for $i \geq k$, we obtain from condition (14) that

$$
s d\left(f x_{n_{i}+1}, T t\right)<\frac{3}{4} d(f t, T t) .
$$

By condition (20), for $i \geq k$, we get

$$
d(f t, T t) \leq s\left[d\left(f t, f x_{n_{i}+1}\right)+d\left(f x_{n_{i}+1}, T t\right)\right]<s d\left(f t, f x_{n_{i}+1}\right)+\frac{3}{4} d(f t, T t) .
$$

Taking limit as $i \rightarrow \infty$, we have

$$
d(f t, T t) \leq \frac{3}{4} d(f t, T t)<d(f t, T t)
$$

which is a contradiction. Therefore, $d(f t, T t)=0$. Since $T t$ is closed, it follows that $u=f t \in$ $T t$, i.e., $u$ is a point of coincidence of $f$ and $T$.

Corollary 1. Let $(X, d)$ be a complete b-metric space with the coefficient $s \geq 1$ and let $G=(V(G), E(G))$ be a graph. Assume that $T: X \rightarrow C B(X)$ is edge preserving and there exist a function $F \in \mathbb{F}_{s}$ which is continuous from right and $\tau>0$ such that

$$
2 \tau+F(s H(T x, T y)) \leq F\left(M_{s}(x, y)\right),
$$

for all $x, y \in X$ with $T x \neq T y$ and $(x, y) \in E(\tilde{G})$ where

$$
M_{s}(x, y)=\max \left\{d(x, y), d(x, T x), \frac{d(y, T y)}{2 s}, \frac{d(x, T y)+d(y, T x)}{2 s}\right\} .
$$

Suppose also that the triple $(X, d, G)$ has the following property:

$(* *)$ If $\left(x_{n}\right)$ is a sequence in $X$ such that $x_{n} \rightarrow x$ and $\left(x_{n}, x_{n+1}\right) \in E(\tilde{G})$ for all $n \geq 1$, then there exists a subsequence $\left(x_{n_{i}}\right)$ of $\left(x_{n}\right)$ such that $\left(x_{n_{i}}, x\right) \in E(\tilde{G})$ for all $i \geq 1$.

If there exists $x_{0} \in X$ such that $\left(x_{0}, z\right) \in E(\tilde{G})$ for some $z \in T x_{0}$, then $T$ has a fixed point in $X$.

Proof. The proof follows from Theorem 3 by taking $f=I$, the identity map on $X$.

Corollary 2. Let $(X, d)$ be a $b$-metric space with the coefficient $s \geq 1$. Let $T: X \rightarrow C B(X)$ and $f: X \rightarrow X$ be such that $T(X) \subseteq f(X)$ and $f(X)$ a complete subspace of $X$. Assume that there exist a function $F \in \mathbb{F}_{s}$ which is continuous from right and $\tau>0$ such that

$$
2 \tau+F(s H(T x, T y)) \leq F\left(M_{s}(f x, f y)\right)
$$

for all $x, y \in X$ with $T x \neq T y$. Then $f$ and $T$ have a point of coincidence in $f(X)$. 
Proof. The proof follows from Theorem 3 by taking $G=G_{0}$, where $G_{0}$ is the complete graph $(X, X \times X)$.

The following corollary is a generalization of Theorem 3.4([15]).

Corollary 3. Let $(X, d)$ be a complete $b$-metric space with the coefficient $s \geq 1$ and let $T: X \rightarrow C B(X)$ be a multi-valued mapping. Assume that there exist a function $F \in \mathbb{F}_{s}$ which is continuous from right and $\tau>0$ such that

$$
2 \tau+F(s H(T x, T y)) \leq F\left(M_{s}(x, y)\right)
$$

for all $x, y \in X$ with $T x \neq T y$. Then $T$ has a fixed point in $X$.

Proof. The proof follows from Theorem 3 by taking $f=I$ and $G=G_{0}$.

Corollary 4. Let $(X, d, \preceq)$ be a partially ordered $b$-metric space with the coefficient $s \geq 1$. Let $T: X \rightarrow C B(X)$ and $f: X \rightarrow X$ be such that $T(X) \subseteq f(X)$ and $f(X)$ a complete subspace of $X$ with the property that if $x, y \in X$ and $f x, f y$ are comparable, then $z_{1}, z_{2}$ are comparable for all $z_{1} \in T x, z_{2} \in T y$. Assume that there exist a function $F \in \mathbb{F}_{s}$ which is continuous from right and $\tau>0$ such that

$$
2 \tau+F(s H(T x, T y)) \leq F\left(M_{s}(f x, f y)\right)
$$

for all $x, y \in X$ with $f x \preceq f y$ or $f y \preceq f x$ and $T x \neq T y$. Suppose also that the triple $(X, d, \preceq)$ has the following property:

If $\left(f x_{n}\right)$ is a sequence in $X$ such that $f x_{n} \rightarrow x$ and $f x_{n}, f x_{n+1}$ are comparable for all $n \geq 1$, then there exists a subsequence $\left(f x_{n_{i}}\right)$ of $\left(f x_{n}\right)$ such that $f x_{n_{i}}, x$ are comparable for all $i \geq 1$.

If there exists $x_{0} \in X$ such that $f x_{0}, z$ are comparable for some $z \in T x_{0}$, then $f$ and $T$ have a point of coincidence in $f(X)$.

Proof. The proof can be obtained from Theorem 3 by taking $G=G_{2}$, where the graph $G_{2}$ is defined by $E\left(G_{2}\right)=\{(x, y) \in X \times X: x \preceq y$ or $y \preceq x\}$.

Corollary 5. Let $(X, d)$ be a complete $b$-metric space with the coefficient $s \geq 1$. Let $\rho$ be a binary relation over $X$ and let $S=\rho \cup \rho^{-1}$. Suppose $T: X \rightarrow C B(X)$ is $S$-preserving and there exist a function $F \in \mathbb{F}_{s}$ which is continuous from right and $\tau>0$ such that

$$
2 \tau+F(s H(T x, T y)) \leq F\left(M_{s}(x, y)\right)
$$

for all $x, y \in X$ with $T x \neq T y$ and $x S y$. Suppose also that the following conditions hold:

(i) $(X, d, S)$ is regular;

(ii) there exists $x_{0} \in X$ such that $x_{0} S z$ for some $z \in T x_{0}$.

Then $T$ has a fixed point in $X$.

Proof. The proof follows from Theorem 3 by taking $f=I$ and $G=(V(G), E(G))$, where $V(G)=X, E(G)=\{(x, y) \in X \times X: x S y\} \cup \triangle$.

As an application of Theorem 3, we obtain the following theorems. 
Theorem 4. Let $(X, d)$ be a $b$-metric space with the coefficient $s \geq 1$ and let $T: X \rightarrow$ $C B(X)$ and $f: X \rightarrow X$ be a hybrid pair of mappings such that $T(X) \subseteq f(X)$ and $f(X)$ a complete subspace of $X$. Assume that there exists $k \in(0,1)$ such that

$$
s H(T x, T y) \leq k M_{s}(f x, f y)
$$

for all $x, y \in X$. Then $f$ and $T$ have a point of coincidence in $f(X)$.

Proof. We take $G=G_{0}=(X, X \times X)$. Let $\tau>0$ be such that $k=e^{-2 \tau}$.

For $x, y \in X$ with $T x \neq T y$, we get from condition (21) that

$$
F(s H(T x, T y)) \leq-2 \tau+F\left(M_{s}(f x, f y)\right),
$$

which gives that $2 \tau+F(s H(T x, T y)) \leq F\left(M_{s}(f x, f y)\right)$, where $F(x)=\ln x$. Thus, all the hypotheses of Theorem 3 hold true and the conclusion of Theorem 4 can be obtained from Theorem 3.

The result stated below is a generalization of Nadler's fixed point theorem [13].

Corollary 6. Let $(X, d)$ be a $b$-metric space with the coefficient $s \geq 1$ and let $T: X \rightarrow$ $C B(X)$ and $f: X \rightarrow X$ be a hybrid pair of mappings such that $T(X) \subseteq f(X)$ and $f(X)$ a complete subspace of $X$. Assume that there exists $k \in(0,1)$ such that

$$
s H(T x, T y) \leq k d(f x, f y)
$$

for all $x, y \in X$. Then $f$ and $T$ have a point of coincidence in $f(X)$.

Proof. As $d(f x, f y) \leq M_{s}(f x, f y)$ for all $x, y \in X$, the result follows from Theorem 4 .

Theorem 5. Let $(X, d)$ be a complete $b$-metric space with the coefficient $s \geq 1$ and let $T: X \rightarrow C B(X)$ be a multi-valued mapping such that

$$
s H(T x, T y) \leq a_{1} d(x, y)+a_{2} d(x, T x)+a_{3} d(y, T y)+a_{4}[d(x, T y)+d(y, T x)]
$$

for all $x, y \in X$, where $a_{1}, a_{2}, a_{3}, a_{4}>0$ and $a_{1}+a_{2}+a_{3}+2 s a_{4}<1$. Then $T$ has a fixed point in $X$.

Proof. Condition (23) gives that

$$
s H(T x, T y) \leq\left(a_{1}+a_{2}+a_{3}+2 s a_{4}\right) M_{s}(x, y)
$$

for all $x, y \in X$, where $0<a_{1}+a_{2}+a_{3}+2 s a_{4}<1$. Taking $k=a_{1}+a_{2}+a_{3}+2 s a_{4}$, it follows from condition (24) that

$$
s H(T x, T y) \leq k M_{s}(x, y)
$$

for all $x, y \in X$, where $k \in(0,1)$ is a constant.

Let $\tau>0$ be such that $k=e^{-2 \tau}$.

For $x, y \in X$ with $T x \neq T y$, we get from condition (25) that

$$
2 \tau+F(s H(T x, T y)) \leq F\left(M_{s}(x, y)\right)
$$

where $F(x)=\ln x$. Taking $G=G_{0}=(X, X \times X)$ and $f=I$, all the hypotheses of Theorem 3 hold true. Thus, Theorem 3 ensures that $T$ has a fixed point in $X$. 
We now present Nadler's fixed point theorem in $b$-metric spaces [13].

Theorem 6. Let $(X, d)$ be a complete b-metric space with the coefficient $s \geq 1$ and let $T: X \rightarrow C B(X)$ be a multivalued mapping. Assume that there exists $k \in(0,1)$ such that

$$
s H(T x, T y) \leq k d(x, y)
$$

for all $x, y \in X$. Then $T$ has a fixed point in $X$.

Proof. Condition (26) implies that

$$
s H(T x, T y) \leq k d(x, y) \leq k M_{s}(x, y)
$$

for all $x, y \in X$, where $k \in(0,1)$ is a constant. Let $\tau>0$ be such that $k=e^{-2 \tau}$.

For $x, y \in X$ with $T x \neq T y$, we get from condition (27) that

$$
2 \tau+F(s H(T x, T y)) \leq F\left(M_{s}(x, y)\right),
$$

where $F(x)=\ln x$. Taking $G=G_{0}=(X, X \times X)$ and $f=I$, we have all the hypotheses of Theorem 3 . Thus, applying Theorem 3 we can obtain a fixed point of $T$.

Remark 2. It is valuable to note that Theorem 3 is a proper generalization (see Example 5) of some multi-valued fixed point theorems including Nadler's fixed point theorem for multivalued mappings ([13]).

Remark 3. Several special cases of Theorem 3 can be obtained by restricting $T: X \rightarrow X$ and taking different $F \in \mathbb{F}_{s}$ and $G$.

The following example shows that Theorem 3 is an extension of Corollary 6 .

Example 5. Let $X=[0, \infty)$ with $d(x, y)=|x-y|^{2}$ for all $x, y \in X$. Then $(X, d)$ is a complete $b$-metric space with $s=2$. Let $G$ be a digraph such that $V(G)=X$ and $E(G)=\Delta \cup\left\{\left(0, \frac{1}{n}\right): n=1,2,3, \cdots\right\}$. Let $T: X \rightarrow C B(X)$ be defined by

$$
T x= \begin{cases}\left\{0, \frac{x}{4}\right\}, & \text { if } 0 \leq x<1 \\ \{0\}, & \text { if } x=1 \\ {\left[x^{2}, x^{2}+5\right],} & \text { if } x>1\end{cases}
$$

and $f x=4 x$ for all $x \in X$. Obviously, $T(X) \subseteq f(X)=X$.

For $x=0, y=2$, we have $f x=0, f y=8, T x=\{0\}, T y=[4,9]$. Therefore,

$$
s H(T x, T y)=s \max \{16,81\}=162>k d(f x, f y)
$$

for any $k \in(0,1)$ and hence condition (22) of Corollary 6 does not hold. So, we can not apply Corollary 6 to obtain a point of coincidence of $f$ and $T$.

For $x=0, y=\frac{1}{4 n}, n \in \mathbb{N}$, we have $f x=0, f y=\frac{1}{n}, T x=\{0\}, T y=\left\{0, \frac{1}{16 n}\right\}$ and so $(f x, f y) \in E(\tilde{G})$ which implies that $\left(z_{1}, z_{2}\right) \in E(\tilde{G})$ for all $z_{1} \in T x, z_{2} \in T y$. Therefore, $T$ is edge preserving w.r.t. $f$. Obviously, $x_{0}=0 \in X$ and $\left(f x_{0}, z\right) \in E(\tilde{G})$ for some $z \in T x_{0}$. 
Moreover, for $x=0, y=\frac{1}{4 n}, n \in \mathbb{N}$, we have $s H(T x, T y)=\frac{s}{256 n^{2}}=\frac{1}{128 n^{2}}$ and

$$
\begin{gathered}
M_{s}(f x, f y)=\max \left\{\begin{array}{l}
d(f x, f y), d(f x, T x), \frac{d(f y, T y)}{2 s}, \\
\frac{d(f x, T y)+d(f y, T x)}{2 s}
\end{array}\right\}= \\
=\max \left\{\begin{array}{l}
d\left(0, \frac{1}{n}\right), d(0,\{0\}), \frac{1}{4} d\left(\frac{1}{n},\left\{0, \frac{1}{16 n}\right\}\right), \\
\frac{d\left(0,\left\{0, \frac{1}{16 n}\right\}\right)+d\left(\frac{1}{n},\{0\}\right)}{2 s}
\end{array}\right\}=\max \left\{\frac{1}{n^{2}}, 0, \frac{225}{1024 n^{2}}, \frac{1}{4 n^{2}}\right\}=\frac{1}{n^{2}} .
\end{gathered}
$$

Thus,

$$
s H(T x, T y)=\frac{1}{128 n^{2}}=\frac{1}{128} M_{s}(f x, f y)<\frac{1}{4} M_{s}(f x, f y)
$$

for all $x, y \in X$ with $(f x, f y) \in E(\tilde{G})$ and $T x \neq T y$. Let $\tau>0$ be such that $\frac{1}{4}=e^{-2 \tau}$. Then, we get from condition (28) that $2 \tau+F(s H(T x, T y))<F\left(M_{s}(f x, f y)\right)$, for all $x, y \in X$ with $(f x, f y) \in E(\tilde{G})$ and $T x \neq T y$, where $F(x)=\ln x$.

Also, any sequence $\left(f x_{n}\right)$ with the property $\left(f x_{n}, f x_{n+1}\right) \in E(\tilde{G})$ must be either a constant sequence or a sequence of the following form

$$
f x_{n}= \begin{cases}0, & \text { if } n \text { is odd } \\ \frac{1}{n}, & \text { if } n \text { is even }\end{cases}
$$

where the words 'odd' and 'even' are interchangeable. Consequently it follows that property (*) holds. Thus, all the hypotheses of Theorem 3 hold true. Then the existence of a point of coincidence of $f$ and $T$ follows from Theorem 3 .

It should be noticed that Theorem 3 can not assure the uniqueness of a point of coincidence. It is obvious that $f$ and $T$ have infinitely many points of coincidence in $f(X)$. In fact, for every $x \in[2,4], f x$ is a point of coincidence of $f$ and $T$.

The following example shows that Theorem 3 remains invalid without property $(*)$.

Example 6. Let $X=[0, \infty)$ with $d(x, y)=|x-y|^{2}$ for all $x, y \in X$. Then $(X, d)$ is a complete $b$-metric space with $s=2$. Let $G$ be a digraph such that $V(G)=X$ and $E(G)=\Delta \cup\{(x, y):(x, y) \in(0,1] \times(0,1], x \leq y\}$. Let $T: X \rightarrow C B(X)$ be defined by

$$
T x= \begin{cases}\{1\}, & \text { if } x=0 \\ \left\{\frac{x}{6}\right\}, & \text { if } x \neq 0\end{cases}
$$

and $f x=\frac{x}{2}$ for all $x \in X$. Obviously, $T(X) \subseteq f(X)=X$.

For $x, y \in X, x \neq y$ and

$$
\begin{gathered}
(f x, f y) \in E(\tilde{G}) \Rightarrow x \neq y, x \neq 0, y \neq 0 \text { and } f x \leq 1, f y \leq 1 \\
\Rightarrow 0<x \leq 2,0<y \leq 2, x \neq y \\
\Rightarrow T x=\left\{\frac{x}{6}\right\}, T y=\left\{\frac{y}{6}\right\}, 0<x, y \leq 2, x \neq y \Rightarrow\left(\frac{x}{6}, \frac{y}{6}\right) \in E(\tilde{G}) .
\end{gathered}
$$

This shows that $T$ is edge preserving w.r.t. $f$. Obviously, $x_{0}=1 \in X$ and $\left(f x_{0}, z\right) \in E(\tilde{G})$ for some $z \in T x_{0}$. 
Further, for $x, y \in X,(f x, f y) \in E(\tilde{G})$ with $T x \neq T y$, we have $x \neq y, 0<x, y \leq$ 2, $T x=\left\{\frac{x}{6}\right\}, T y=\left\{\frac{y}{6}\right\}$. Therefore,

$$
s H(T x, T y)=s d\left(\frac{x}{6}, \frac{y}{6}\right)=\frac{1}{18}|x-y|^{2}=\frac{2}{9} d(f x, f y)<\frac{4}{9} d(f x, f y) \leq \frac{4}{9} M_{s}(f x, f y)
$$

for all $x, y \in X,(f x, f y) \in E(\tilde{G})$ with $T x \neq T y$. Let $\tau>0$ be such that $\frac{4}{9}=e^{-2 \tau}$. Then, we get from condition (29) that

$$
2 \tau+F(s H(T x, T y))<F\left(M_{s}(f x, f y)\right),
$$

for all $x, y \in X$ with $(f x, f y) \in E(\tilde{G})$ and $T x \neq T y$, where $F(x)=\ln x$. Thus, condition (1) of Theorem 3 holds true. But property $(*)$ does not hold true. In fact, if we consider the sequence $\left(f x_{n}\right)$ where $x_{n}=\frac{2}{n}$, then $f x_{n} \rightarrow 0$ and $\left(f x_{n}, f x_{n+1}\right) \in E(\tilde{G})$ for all $n \in \mathbb{N}$. But there exists no subsequence $\left(f x_{n_{i}}\right)$ of $\left(f x_{n}\right)$ such that $\left(f x_{n_{i}}, 0\right) \in E(\tilde{G})$. Thus, all the hypotheses of Theorem 3 hold true except property $(*)$. As a result, we observe that $f$ and $T$ have no point of coincidence in $X$.

Acknowledgements. The authors would like to thank the referees for their valuable comments.

\section{REFERENCES}

1. J. Ahmad, A. Al-Rawashdeh, A. Azam, New fixed point theorems for generalized F-contractions in complete metric spaces, Fixed Point Theory and Applications, 80 (2015).

2. I. Altun, G. Durmaz, G. Minak, S. Romaguera, Multivalued Almost F-contractions on complete metric spaces, Filomat, 30 (2016), 441-448.

3. V. Berinde, Generalized contractions in quasimetric spaces, In: Seminar on Fixed Point Theory, (1993), $3-9$.

4. M. Berinde, V. Berinde, On a general class of multi-valued weakly Picard mappings, J. Math. Anal. Appl., 326 (2007), 772-782.

5. I.A. Bakhtin, The contraction mapping principle in almost metric spaces, Funct. Anal., Gos. Ped. Inst. Unianowsk, 30 (1989), 26-37.

6. S. Banach, Sur les opérations dans les ensembles abstraits et leur application aux équations intégrales, Fund. Math., 3 (1922), 133-181.

7. J.A. Bondy, U.S.R. Murty, Graph theory with applications, American Elsevier Publishing Co., Inc., New York, 1976.

8. I. Beg, A.R. Butt, S. Radojevic, The contraction principle for set valued mappings on a metric space with a graph, Comput. Math. Appl., 60 (2010), 1214-1219.

9. F. Bojor, Fixed point of $\varphi$-contraction in metric spaces endowed with a graph, Annala of the University of Cralova, Mathematics and Computer Science Series, 37 (2010), 85-92.

10. F. Bojor, Fixed points of Kannan mappings in metric spaces endowed with a graph, An. St. Univ. Ovidius Constanta, 20 (2012), 31-40.

11. M. Boriceanu, Strict fixed point theorems for multivalued operators in b-metric spaces, Int. J. Mod. Math., 4 (2009), 285-301.

12. S. Czerwik, Contraction mappings in b-metric spaces, Acta Math. Inform. Univ. Ostrav, 1 (1993), 5-11.

13. S. Czerwik, Nonlinear set-valued contraction mappings in b-metric spaces, Atti Semin. Mat. Fis. Univ. Modena, 46 (1998), 263-276.

14. S. Czerwik, K. Dlutek, SL. Singh, Round-off stability of iteration procedures for set-valued operators in b-metric spaces, J. Natur. Phys. Sci., 11 (2007), 87-94. 
15. M. Cosentino, M. Jleli, B. Samet, C. Vetro, Solvability of integrodifferential problems via fixed point theory in b-metric spaces, Fixed Point Theory and Applications, 70 (2015).

16. G. Chartrand, L. Lesniak, P. Zhang, Graph and digraph, CRC Press, New York, NY, USA, 2011.

17. F. Echenique, A short and constructive proof of Tarski's fixed point theorem, Internat. J. Game Theory, 33 (2005), 215-218.

18. R. Espinola, W.A. Kirk, Fixed point theorems in R-trees with applications to graph theory, Topology Appl., 153 (2006), 1046-1055.

19. Y. Feng, S. Liu, Fixed point theorems for multi-valued contractive mappings and multi-valued Caristi type mappings, J. Math. Anal. Appl., 317 (2006), 103-112.

20. J.I. Gross, J. Yellen, Graph theory and its applications, CRC Press, New York, NY, USA, 1999.

21. M.E. Gordji, H. Baghani, H. Khodaei, M. Ramezani, A generalization of Nadler's fixed point theorem, The Journal of Nonlinear Science and Applications, 3 (2010), 148-151.

22. N. Hussain, R. Saadati, R.P. Agrawal, On the topology and wt-distance on metric type spaces, Fixed Point Theory and Applications, 88 (2014).

23. J. Jachymski, The contraction principle for mappings on a metric space with a graph, Proc. Amer. Math. Soc., 136 (2008), 1359-1373.

24. H. Kaneko, S. Sessa, Fixed point theorems for compatible multi-valued and single-valued mappings, Internat. J. Math. and Math. Sci., 12 (1989), 257-262.

25. S.K. Mohanta, Common fixed points in b-metric spaces endowed with a graph, Matematicki Vesnik, 68 (2016), 140-154.

26. S.K. Mohanta, Fixed Points in $C^{*}$-algebra valued b-metric spaces endowed with a Graph, Mathematica Slovaca, 68 (2018), 639-654.

27. S.K. Mohanta, S. Patra, Coincidence points and common fixed points for hybrid pair of mappings in b-metric spaces endowed with a graph, J. Lin. Top. Alg., 6 (2017), 301-321.

28. S. Nadler, Multi-valued contraction mappings, Pac. J. Math., 20 (1969), 475-488.

29. H.K. Pathak, Fixed point theorems for weak compatible multi-valued and single-valued mappings, Acta Math. Hungar, 67 (1995), 69-78.

30. J. Tiammee, S. Suantal, Coincidence point theorems for graph-preserving multi-valued mappings, Fixed Point Theory and Applications, 70 (2014).

31. D. Wardowski, Fixed points of a new type of contractive mappings in complete metric spaces, Fixed Point Theory and Applications, 94 (2012).

Department of Mathematics, West Bengal State University

Barasat, 24 Parganas (North), West Bengal, India

${ }^{1}$ smwbes@yahoo.in; ${ }^{2}$ ratulkar1@gmail.com 